\title{
Haplotype-level metabarcoding of freshwater macroinvertebrate species: a prospective tool for population genetic analysis
}

\author{
Joeselle Serrana ${ }^{1}$ and Kozo Watanabe ${ }^{2}$ \\ ${ }^{1}$ Center for Marine Environmental Studies, Ehime University \\ ${ }^{2}$ Ehime University
}

October 26, 2021

\begin{abstract}
The development and evaluation of DNA metabarcoding protocols for haplotype-level resolution require attention, specifically for population genetic analysis, i.e., parallel estimation of genetic diversity and dispersal patterns among multiple species present in a bulk sample. Further exploration and assessment of the laboratory and bioinformatics strategies are warranted to unlock the potential of metabarcoding-inferred population genetic analysis. Here, we assessed the inference of freshwater macroinvertebrate haplotypes from DNA metabarcoding data using mock samples with known Sanger-sequenced haplotypes. We also examined the influence of different DNA template concentrations and PCR cycles on detecting true haplotypes and the reduction of spurious haplotypes obtained from DNA metabarcoding. We tested our haplotyping strategy on a mock sample containing 20 specimens from four species with known haplotypes based on the 658-bp Folmer region of the mitochondrial cytochrome c oxidase gene. The read processing and denoising step resulted in 14 zero-radius operational taxonomic units (ZOTUs) of 421-bp length, with 12 ZOTUs having $100 \%$ match with 12 of the Sanger haplotype sequences. Quality passing reads relatively increased with increasing PCR cycles, and the relative abundance of each ZOTUs was consistent for each cycle number. This suggests that increasing the cycle number from 24 to 64 did not affect the relative abundance of quality passing filter reads of each ZOTUs. Our study demonstrated the ability of DNA metabarcoding to infer intraspecific variability while highlighting the challenges that need to be addressed before its possible applications to population genetic studies.
\end{abstract}

\section{Hosted file}

MS-Meta_Inf_Haplotyping-Main.docx available at https://authorea.com/users/390531/articles/ 543136-haplotype-level-metabarcoding-of-freshwater-macroinvertebrate-species-aprospective-tool-for-population-genetic-analysis 